\title{
Realistic examples of chaotic magnetic fields created by wires
}

\author{
J. Aguirre ${ }^{1(a)}$ and D. Peralta-Salas ${ }^{2(b)}$ \\ ${ }^{1}$ Centro de Astrobiología, CSIC-INTA - Ctra. Ajalvir, km 4, 28850 Torrejón de Ardoz, Madrid, Spain \\ ${ }^{2}$ Departamento de Matemáticas, Universidad Carlos III de Madrid - 28911 Leganés, Spain
}

received 13 June 2007; accepted in final form 21 October 2007

published online 13 November 2007

PACS 05.45.Ac - Low-dimensional chaos

PACS 41.20.Gz - Magnetostatics; magnetic shielding, magnetic induction, boundary-value problems

\begin{abstract}
In this paper we study some examples of magnetic fields with hyperbolic periodic orbits and erratic behavior. The remarkable fact is that the fields under consideration are originated by configurations which consist of two thin wires. We present a rigorous proof of the chaoticity of these magnetic fields, in the sense that they possess KAM islands and homoclinic tangles, and we also provide numerical simulations. In particular we illustrate, contrary to folk wisdom, that magnetic lines originated by current filaments can show a very complex nature. Finally, we propose a simple experimental verification of these results, as well as possible fields of application.
\end{abstract}

Copyright (C) EPLA, 2007

Introduction. - Magnetic fields created by current distributions usually appear in physics, both in theory and applications. The most suitable approach is the dynamical systems one, i.e. visualizing the magnetic field $B$ as a vector field in $\mathbb{R}^{3}$, the orbits of $B$ being called magnetic lines. This viewpoint goes back to Faraday and is important in multidisciplinary research, e.g. biomedical engineering [1], electrical engineering [2], spectroscopy [3] and medical applications as magnetic resonance imaging [4]. In most applications the configurations of wires possess some Euclidean symmetry, e.g. rotational symmetry [5].

In general it is very difficult to get closed analytical expressions for the magnetic fields originated by wires, see [5]. For this reason the qualitative theory of dynamical systems is an effective tool in order to study the most relevant properties of the magnetic lines, as well as their action on charged particles, without actually integrating the Biot-Savart law [6].

In [7] and [8] the symmetries and first integrals of magnetic fields originated by certain current distributions were studied. It was proved that charged particles subjected to these fields verify the non-swallowing property, i.e. they cannot approach the wires indefinitely. The phase portraits of the fields were also described. In [8] it was posed the major unanswered question of finding

\footnotetext{
(a) E-mail: aguirreaj@inta.es

(b) E-mail: dperalta@math.uc3m.es
}

a configuration of wires giving rise to a chaotic (nonintegrable) magnetic field.

Let us briefly comment on the contexts in which chaotic magnetic fields have already been obtained. Examples of divergence-free chaotic dynamical systems were found in [9], but these examples are not realistic. Chaotic magnetic lines have also been described in the divertor region when analyzing tokamak dynamics in the context of plasma physics, but these magnetic fields are not created by wires. Indeed the divertor region is sometimes modelled by thin wires, see e.g. $[10,11]$, which give rise to a non-chaotic portrait (the configuration has some Euclidean symmetry). Chaos appears when a vacuum field perturbation is added to the system, but the source of this perturbation is not a current distribution. In some cases the perturbation is modelled by a complicated structure of wires or a surface current density (this is called ergodic limiter in the specialized literature, see $[12,13])$, but the final field, which is chaotic, is in the tokamak region and hence part of it is created by a plasma current density. Apart from plasma physics (tokamaks) [14,15] chaotic magnetic lines have also appeared in other parts of physics, e.g. coronal structures [16] and force-free fields [17], although, of course, these fields are not created by wires and possess chaotic sources.

Physicists and engineers have widely believed that chaos is not possible when the magnetic field is created by few thin wires. In fact the wrong idea that magnetic lines produced by current filaments form closed loops is 
expressed in many standard textbooks, as Stratton [18] in chapt. 4.1, p. 225, Reitz-Milford [19] in sect. 10-7, p. 201, Jefimenko [20] in chapt. 10-4, p. 328, Sadiku [21] in chapt. 7.5, p. 311 and Tipler [22] in sect. 29-3, p. 897. The first one in suggesting that this belief could be wrong was Ulam [23], but he did not provide any example. The only paper available about this subject, as far as we know, is [24], where Morrison provided some configurations formed by two wires giving rise to chaotic magnetic fields. However, a proof of this claim was not given, and numerical simulations were not carefully explained. In fact the author worked under the assumption that one of the current intensities is much bigger than the other.

In this paper we study the configurations of wires introduced by [24], that is, a straight-line wire and a circular wire in axisymmetric position, or small perturbations of it. We provide a mathematical proof of the existence of chaos and detailed numerical results on the Poincaré map, the structure of magnetic lines and the extension of the chaotic region. As a spin-off we prove that these magnetic fields possess isolated periodic orbits with stable and unstable invariant manifolds. The main motivation of this work is twofold: on the one hand, we show that complicated magnetic portraits can be created using few current filaments, contrary to what is widely believed by many physicists and engineers; on the other hand, we report phenomena (periodic, quasi-periodic and stochastic magnetic lines) which have already been observed in magnetic confinement systems and coronal structures, but with the important difference that the source of the chaotic magnetic fields in this work is not a plasma but just two thin cables. The inexpensive and simple way of producing this kind of fields suggests the interest of this phenomenon in applications, as we will discuss in the final section.

Let us summarize the contents of this paper. In the second section we study the magnetic field created by certain current configuration $C$, in particular, the existence of both quasi-periodic and knotted magnetic lines. This study of the unperturbed configuration is key to apply perturbation theorems in the next section. Indeed, we show, analytically in the third section and numerically in the fourth section, that for generic deformations of the distribution there exist quasi-periodic magnetic lines, KAM islands and chaotic (hyperbolic) regions. The main tools that we use are the same as in refs. [15,25], i.e. Poincaré map and KAM, Poincaré-Birkhoff and SmaleBirkhoff theorems [26-28]. Finally, in the fifth section, some ideas concerning applications and experimental verification of these results are included.

For the sake of simplicity, we have assumed the thin-wire model, as is usual in the literature [5-8], although using finite-thickness wires would not change the results. Note that the quasi-periodic, knotted and chaotic orbits that we prove to exist belong to domains separated from the wires, and hence they would not interact with the surfaces of the thick current lines.
In ending this introduction let us make a brief comment on the Hamiltonian structure of divergence-free vector fields. There are many ways to prove that, locally, in neighborhoods of non-singular points, divergencefree vector fields have a Hamiltonian description, see e.g. $[10,11,24,29]$. This fact has been semi-rigorously used since the pioneering works $[30,31]$ to study perturbations and chaos of divergence-free vector fields. Anyway, chaos is a global property of the solutions and it is not immediate at all that results in Hamiltonian dynamics can be applied in general to divergence-free vector fields, as e.g. KAM theorem. This is the reason why rigorous general mathematical results on this subject are very hard to obtain, see e.g. [32-35]. Furthermore, when working with the specific class of vector fields obtained through Biot-Savart law from thin wires, it is not known whether these general results for divergence-free systems still hold. In our particular examples we do not need so much because it is possible to reduce the problem to area-preserving maps and hence we can apply standard KAM, Poincaré-Birkhoff and Birkhoff-Smale theorems.

Knots and quasi-periodic orbits. - Let $C$ be the current distribution formed by a straight-line wire $L_{1}=\{z-$ axis $\}$ and a circular wire $L_{2}$ on the $x y$-plane, centered at the origin and of unit radius. Assume that the wires carry the same constant current $J$, although this hypothesis is not crucial and all the results in this paper hold if the wires carry different intensities. In order to simplify the mathematical expressions let us set $\frac{\mu_{0} J}{4 \pi}=1$.

As usual $\mathbb{R}^{3}$ is endowed with Cartesian coordinates $(x, y, z)$ and Cylindrical coordinates $(r, \phi, z)$. The orthonormal basis in cylindrical coordinates is denoted by $\left\{u_{r}, u_{\phi}, u_{z}\right\}$. Every distance is measured in meters.

The magnetic field $B$ induced by $C$ is the sum of the separate contributions of $L_{1}$ (magnetic field $B_{1}$ ) and $L_{2}$ (magnetic field $B_{2}$ ), and it has the expression [8]

$$
B=B_{1}+B_{2}=\frac{2 u_{\phi}}{r}+B_{r}(r, z) u_{r}+B_{z}(r, z) u_{z} .
$$

The components $B_{r}$ and $B_{z}$ can be expressed in terms of elliptic functions [8], but this is not relevant for our purposes.

The magnetic field $B$ has a first integral $I(r, z)$ whose level sets (magnetic surfaces) resemble revolution tori around $L_{2}$. In [8] it was proved that the function $I(r, z)$ is decreasing from $L_{2}$ and tends to zero at infinity and on $L_{1}$. Note now that the orbits of the vector field $\frac{1}{2} r^{2} B_{1}=r u_{\phi}$ have constant period $T_{1}=2 \pi$ and the orbits of $\frac{1}{2} r^{2} B_{2}$ have a period which is a non-constant function of $I, T_{2}=F(I)$. This implies that the rotation number

$$
\frac{T_{1}}{T_{2}}=\frac{2 \pi}{F(I)}
$$

is non-constant. On the tori $I=c$ for which $T_{1} / T_{2} \in \mathbb{Q}$ the orbits of $B$ are periodic (note that the lines of $B$ and $\frac{1}{2} r^{2} B$ 
coincide). Otherwise the orbits of $B$ are quasi-periodic, i.e. dense on $I=c$.

Furthermore, when $\frac{T_{1}}{T_{2}}=\frac{m}{n}$ the magnetic lines on $I=c$ make $m$ turns around the circular wire while they wrap $n$ times in the longitudinal direction, thus showing that they give rise to $m-n$ torus knots [27].

This simple configuration illustrates that magnetic lines do not need to be simple at all. In particular, they do not need to be closed and even the periodic ones can be nontrivially knotted. This suggests how complex the magnetic lines can become when considering more involved configurations of wires (e.g., breaking the axisymmetry). The reader must keep in mind the results in this section when passing to the next section, where perturbations of the configuration $C$ are introduced. The reason is that the structure of the unperturbed field is very important in order to apply perturbation techniques.

Hyperbolic cycles and chaos. - Perfect straight-line or circular wires do not appear in practical situations, for this reason in this section we work with a new configuration of wires $C^{\epsilon}$ which is a small perturbation of $C\left(\lim _{\epsilon \rightarrow 0} C^{\epsilon}=C\right)$. This small deformation is arbitrary when considering $L_{2}$ but it must satisfy certain bound conditions when considering $L_{1}$ (because of its noncompactness) [8]. The magnetic field $B_{\epsilon}$ associated to $C^{\epsilon}$ is therefore a small perturbation of $B$, that is

$$
B_{\epsilon}=B+O(\epsilon),
$$

in certain solid torus $K$ containing the circular wire, but not very close to the wires. Equation (2) is essential in order to apply classical perturbation theorems of dynamical systems. In what follows we will assume that the reader is familiar with the standard tools and arguments in KAM, Poincaré-Birkhoff and Birkhoff-Smale theorems. Details of these theories can be consulted, e.g. in [26-28].

Let us now construct the Poincaré map $P_{0}$ associated to $B$. Consider the annulus $A=I^{-1}\left[c_{1}, c_{2}\right] \cap\{\phi=0\}$, where $I^{-1}\left[c_{1}, c_{2}\right] \subset K$ is the solid region around the circular wire $L_{2}$ bounded by the tori $I^{-1}\left(c_{1}\right)$ and $I^{-1}\left(c_{2}\right)$. Let us endow $A$ with local coordinates $(I, \theta) . P_{0}$ is defined as $P_{0}:(I, \theta) \in$ $A \longrightarrow(I, \theta+h(I)(\bmod 2 \pi))=\left(I, \theta+\frac{2 \pi m}{n}(\bmod 2 \pi)\right) \in A$. It is clear that $P_{0}$ is a twist map [27].

The Poincaré map $P_{\epsilon}$ associated to $B_{\epsilon}$ is a small perturbation of $P_{0}$ and it is area preserving because $B_{\epsilon}$ is divergence free. Since the period $F(I)$ is non-constant, the non-degeneracy condition is satisfied and hence the KAM theorem can be applied to a neighborhood of each torus in $I^{-1}\left[c_{1}, c_{2}\right] \subset K[26,27]$. This result on the Poincaré map implies that the magnetic field $B_{\epsilon}$ has a set of invariant tori of positive Lebesgue measure $\mu(\epsilon)$ close to the invariant tori of $B$ in $K$. Moreover, $\mu(\epsilon) / \mu(0) \rightarrow 1$ as $\epsilon \rightarrow 0$ and these surviving tori are filled with quasi-periodic orbits (i.e., non-resonant tori). Consequently, most of the quasiperiodic orbits of $B$ in $K$ are preserved, up to deformation, when the configuration $C$ is perturbed. It is also important to note that, as a consequence of the KAM theorem, the map $P_{\epsilon}$ can be defined in a set diffeomorphic to an annulus on the plane $\phi=0$. The boundaries of this annulus are formed by any two irrational invariant tori which are deformed by the perturbation. Without loss of generality this set can be taken to be $A$, up to diffeomorphism.

The most surprising phenomenon does not lie on the irrational tori, but in the regions in between these tori, where chaos is manifested.

Indeed, choose a closed curve $\Gamma_{0}=I^{-1}(c) \cap A$, invariant under $P_{0}$, such that its rotation number is $m / n$. Observe that the period of the orbits increases when we move away from the closed wire, while the value of the first integral decreases. Then it is clear that $\Gamma_{0}$ is formed by fixed points of $P_{0}^{n}$, and that $P_{0}^{n}$ rotates through an angle greater than $2 \pi$ in the invariant curve $I^{-1}\left(c^{\prime}\right) \cap A$ and less than $2 \pi$ in the invariant curve $I^{-1}\left(c^{\prime \prime}\right) \cap A, c^{\prime \prime}<c<c^{\prime}$ and $I^{-1}\left(c^{\prime}\right)$, $I^{-1}\left(c^{\prime \prime}\right)$ are assumed to be non-resonant tori which survive after perturbation. This behavior persists for $P_{\epsilon}^{n}$ and hence there exists a closed curve $\Gamma_{\epsilon}$ which moves only in radial direction under $P_{\epsilon}^{n}$. Note that $\Gamma_{\epsilon} \rightarrow \Gamma_{0}$ as $\epsilon \rightarrow 0$. For a generic perturbation of the wires the curves $P_{\epsilon}^{n}\left(\Gamma_{\epsilon}\right)$ and $\Gamma_{\epsilon}$ are transversal, thus implying, since $P_{\epsilon}^{n}$ preserves area, that $P_{\epsilon}^{n}$ has (at least) $2 n$ fixed points, half-elliptic centers and half-hyperbolic saddles [27].

Consequently, we have proved that for each resonant torus of $B$ in $K$ the magnetic field $B_{\epsilon}$ has (at least) a hyperbolic periodic orbit and an elliptic periodic orbit. Since $B_{\epsilon}$ is divergence free it is clear that the hyperbolic cycles are neither attractors nor repellers and hence they possess stable and unstable invariant manifolds. These invariant manifolds are confined between neighboring irrational tori, the so-called stochastic layers or homoclinic tangles $[27,28]$, and for a generic perturbation they intersect transversally. According to the Birkhoff-Smale theorem the number of intersections is in fact infinite and this implies the presence of horseshoe-type dynamics. Consequently, the magnetic lines behave chaotically in these regions, i.e. there are hyperbolic invariant sets with dense magnetic lines, there do not exist any global analytic first integrals and the highest Lyapunov exponents are positive. On the other hand, the elliptic periodic orbits give rise to very narrow stability regions called KAM islands.

It is important to remark that, as a consequence of KAM, Poincaré-Birkhoff and Birkhoff-Smale theorems, chaos arises for any generic small perturbation of configuration $C$. In the following section, we will illustrate our results with a particular type of perturbation, although almost any other perturbation would give rise to the same phenomena, as we have shown in the analytical proof.

Numerical verification of chaotic magnetic lines. - Several numerical computations have been done to illustrate the analytical results presented in the paper. We have chosen the perturbation as simple as possible although our results hold for generic small perturbations as shown in the third section. The perturbation is applied to the circular wire, say $L_{2}^{\epsilon}$, in a way that the current 


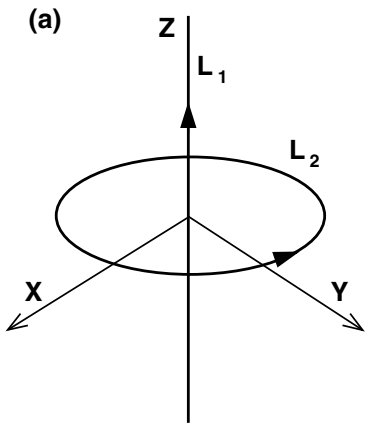

Fig. 1: Diagrams for the wire configuration $C$ (a) and its perturbed version $C^{\epsilon}(\mathrm{b})$. The direction of the currents is marked with arrows. For clarity, the value of the perturbation $\epsilon$ has been strongly enlarged in the picture.

experiences $k$ sinusoidal oscillations of amplitude $\epsilon$ in the $z$-axis (see fig. 1 for a sketch of configuration $C$ and its perturbed version $C^{\epsilon}$ ). The current distribution $C^{\epsilon}$ hence becomes

$$
\begin{aligned}
& C^{\epsilon}=L_{1} \cup L_{2}^{\epsilon}, \\
& L_{1}=\{x=0, y=0\}, \\
& L_{2}^{\epsilon}=\{x=\cos \phi, y=\sin \phi, z=\epsilon \sin (k \phi)\} .
\end{aligned}
$$

We have chosen $k=10$ and $\epsilon=0.05$ and 0.02 for our calculations, but the results are qualitatively independent of this choice of parameters as far as $\epsilon$ is sufficiently small to apply KAM, Poincaré-Birkhoff and BirkhoffSmale theorems.

The numerical resolution of the magnetic lines of this system must be done with some care. In each step of the integration of every magnetic line, the value of the magnetic field must be calculated in a different point of phase space. While the magnetic field created by the vertical wire $L_{1}$ is explicitly known, we need to solve numerically the Biot-Savart law due to the perturbed circular wire $L_{2}^{\epsilon}$. We have done this applying the extended trapezoidal rule for integrating functions [36]. Furthermore, the magnetic field can take relatively high values when the line gets close to the wires, and a short numerical step is required in the computation of the line to avoid numerical errors. A 4th-order RungeKutta method with a step $\Delta=10^{-4}$ is enough for our qualitative purposes, but it is worth mentioning that for double-precision results a higher-order numerical method is advisable [14].

Figure 2(a) shows for $\epsilon=0.05$ the intersection of several numerically computed magnetic lines with the plane $x=0$, in the region where $y>0$ (that is, a Poincaré section). Note that the current $J$ carried by the wires affects the value of the magnetic field $B_{\epsilon}$ but not the shape of its magnetic lines, and therefore this picture is independent of $J$. Magnetic lines of different nature are clearly observable, mainly a chaotic region around $L_{2}^{\epsilon}$ and a countable number of KAM tori surrounding the chaotic sea (other thin chaotic regions were found in between the external KAM

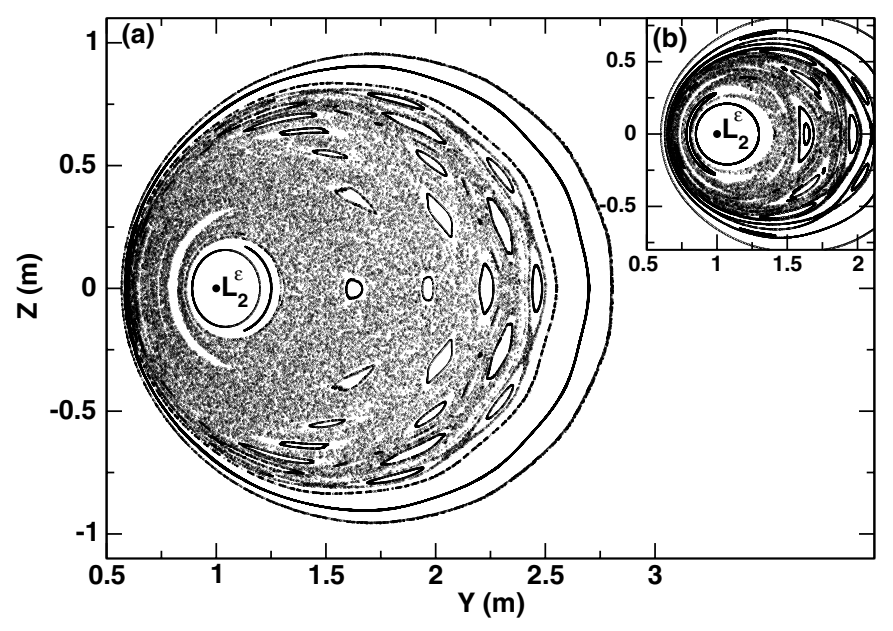

Fig. 2: Poincaré sections of the magnetic field created by the perturbed system $C^{\epsilon}$, when $\epsilon=0.05$ (a) and $\epsilon=0.02$ (b). An extensive chaotic sea containing KAM islands is surrounded by several KAM tori for high values of the perturbation $\epsilon$, but the chaotic region shrinks when $\epsilon \rightarrow 0$. The intersection of the perturbed circular wire $L_{2}^{\epsilon}$ with the plane $x=0$ is remarked in both figures.

tori, but they were not plotted for the sake of clarity). The chaotic region presents several KAM islands, and in each of these little bounded regions elliptic periodic orbits can be found.

When the perturbation $\epsilon$ decreases, the KAM islands grow while the chaotic region shrinks. This is clear in fig. 2(b), where $\epsilon=0.02$. In the limit of $\epsilon=0$, quasiperiodic orbits fill up the whole phase space; we recover then the non-chaotic structure of magnetic lines that corresponds to the infinite straight-line wire $L_{1}$ plus the unperturbed circular wire $L_{2}$ (that is, configuration $C$ shown in fig. 1(a).)

Figure 3 shows three typical magnetic lines obtained for the perturbed system when $\epsilon=0.05$ : fig. 3(a) shows an elliptic periodic line obtained from the interior of a KAM island, fig. 3(b) shows a quasi-periodic line contained in one of the KAM islands and fig. 3(c) shows a part of a chaotic magnetic line. Since the magnetic field $B_{\epsilon}$ is divergence free, the addition of the three Lyapunov exponents $\lambda_{1} \geqslant \lambda_{2} \geqslant \lambda_{3}$ of every magnetic line must also be zero. The numerical computations verify this condition, as well as the fact that $\lambda_{1}=\lambda_{2}=\lambda_{3}=0$ for periodic and quasi-periodic lines, while $\lambda_{1}=-\lambda_{3}>0$ and $\lambda_{2}=0$ for the chaotic lines.

Final remarks. - It would be interesting to detect the quasi-periodic and chaotic magnetic lines of $B_{\epsilon}$ experimentally. The chaotic region is rather extensive even for small perturbations, and the configuration of wires in this paper is very simple. Therefore, we believe that it should not be a difficult task to arrange a suitable experimental device to follow magnetic lines and check whether their nature is regular or chaotic. The experimental set-up would be based on a precise use of magnetometers, in order 

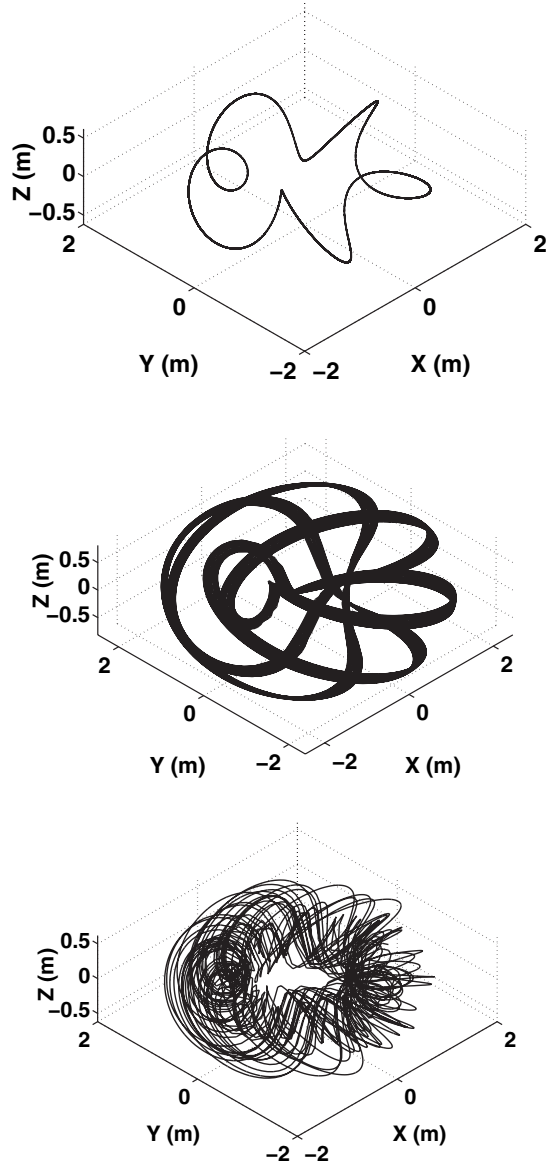

Fig. 3: Plot of three types of magnetic lines obtained in the perturbed system $C^{\epsilon}$, when $\epsilon=0.05$. (a) Elliptic periodic magnetic line. (b) Quasi-periodic magnetic line from a KAM island. (c) Chaotic magnetic line.

to describe the field lines around the current distribution. Let us observe that chaotic portraits created by real long thin wires should be indistinguishable from those created by infinite wires and shown in this paper, as far as the real wire is much longer than the diameter of the circular one. Specifically, the infinite wire can be substituted by a loop wire of radius much bigger than the radius of the circular wire on the $x y$-plane and we should also find chaotic magnetic portraits (theoretically it can be proved that this is indeed the case). Furthermore, any method for driving current in a closed deformed loop is good because chaotic portraits are not restricted to a particular case (e.g., the fourth section) but they arise for generic perturbations. Since the theoretical study is generally quite complicated, different current distributions might be experimentally tested in order to find other configurations with chaotic magnetic lines.

Regarding the practical importance of chaotic or ergodic magnetic lines originated by current distributions let us briefly mention two directions. In the context of plasma physics some applications have been proposed concerning the plasma-wall interaction and the control of plasma contamination [15]. In the context of magnetobiology the effects of magnetic fields on biological systems are object of extensive study [37], and we believe that the interaction of chaotic magnetic fields with biological entities could give rise to interesting phenomena. Observe that these research directions are related to the problem of studying the motion of charged particles in regions filled by chaotic magnetic lines.

$$
* * *
$$

We are very grateful to the referees of this paper for their useful criticisms, which have helped to improve this article. The authors are also grateful to M. ARRAYÁs, S. C. MAnRubia and P. PoKorny for their interesting comments on different parts of this work. DP-S is financially supported by the Spanish Ministry of Education and Science through the Juan de la Cierva program. He also acknowledges the partial support of the DGICYT under grant No. MTM2007-62478.

\section{REFERENCES}

[1] Zborowski M., Midura R. J., Wolfman A., Patterson T., Ibiwoye M., Sakai Y. and Grabiner M., Ann. Biomed. Eng., 31 (2003) 195.

[2] Kiessling F., Nefzger P., Nolasco J. F. and Kaintzyk U., Overhead Power Lines (Springer, New York) 2003.

[3] Bergeman T., Erez G. and Metcalf H. J., Phys. Rev. A, 35 (1987) 1535.

[4] HaAcke E. M., Brown R. W., Venkatesan R. and Thompson M. R., Magnetic Resonance Imaging (Wiley, New York) 1999.

[5] Conway J. T., IEEE Trans. Mag., 37 (2001) 2977.

[6] JACKSON J. D., Classical Electrodynamics (Wiley, New York) 1999.

[7] González-Gascón F. and Peralta-Salas D., Phys. Lett. A, 333 (2004) 72.

[8] González-Gascón F. and Peralta-Salas D., Phys. D, 206 (2005) 109.

[9] Zugasti M. A., Gómez J. M. E., Miró D. G. P., Llorente F. R. and RañAdA A. F., Chaos, Solitons Fractals, 4 (1994) 1943.

[10] Boozer A. H. and Rechester A. B., Phys. Fluids, 21 (1978) 682.

[11] Pomphrey N. and Reiman A., Phys. Fluids B, 4 (1992) 938.

[12] da Silva E. C., Caldas I. L. and Viana R. L., Phys. Plasmas, 8 (2001) 2855.

[13] Pires C. J. A., Saettone E. A. O. and Kucinski M. Y., Plasma Phys. Control. Fusion, 47 (2005) 1609.

[14] Kogoshi S., Terada T. and Maeda J., J. Phys. Soc. Jpn., 67 (1998) 3649.

[15] da Silva E. C., Caldas I. L., Viana R. L. and Sanjuán M. A. F., Phys. Plasmas, 9 (2002) 4917.

[16] Pontin D. I., Priest E. R. and Longcope D. W., Sol. Phys., 212 (2003) 319.

[17] Gilbert A. D. and Childress S., Phys. Rev. Lett., 65 (1990) 2133. 
[18] Stratton J. A., Electromagnetic Theory (McGraw-Hill, New York) 1941.

[19] Reitz J. R. and Milford F. J., Foundations of Electromagnetic Theory (Addison-Wesley, Reading, Mass.) 1967.

[20] Jefimenko O. D., Electricity and Magnetism (Electret Scientific Company, Star City) 1989.

[21] Sadiku M. N. O., Elements of Electromagnetics (Saunders College Publishing, Philadelphia) 1994.

[22] Tipler P. A., Physics for Scientists and Engineers (W. H. Freeman and Company, New York) 1999.

[23] Ulam S. M., Problems in Modern Mathematics (Wiley, New York) 1960.

[24] Morrison P. J., Phys. Plasmas, 7 (2000) 2279.

[25] Ullmann K. and Caldas I. L., Chaos, Solitons Fractals, 11 (2000) 2129.

[26] Arnold V. I., Mathematical Methods of Classical Mechanics (Springer, New York) 1989.

[27] Guckenheimer J. and Holmes P., Nonlinear Oscillations Dynamical Systems and Bifurcations of Vector Fields (Springer, New York) 2002.
[28] Отт E., Chaos in Dynamical Systems (Cambridge University Press, Cambridge) 2002.

[29] Tang X. and Boozer A. H., Phys. Lett. A, 236 (1997) 476.

[30] Kerst D. W., Plasma Phys., 4 (1962) 253.

[31] Rosenbluth M. N., Sagdeev R. Z. and Taylor J. B., Nucl. Fusion, 6 (1966) 297.

[32] Broer H. W., Lect. Notes Math., 898 (1981).

[33] Broer H. W., Huitema G. B., Takens F. and Branksma B. L. J., Mem. Am. Math. Soc. 83 (1990).

[34] Sevryuk M. B., Russ. Math. Surv., 50 (1995) 341.

[35] Broer H. W., Huitema G. B. and Sevryuk M. B., Lect. Notes Math., 1645 (1996)

[36] Press W. H., Flannery B. P., Teukolsky S. A. and Vetterling W. T., Numerical Recipes: The Art of Scientific Computing (Cambridge University Press, Cambridge) 2007.

[37] Binhi V. N., Magnetobiology (Academic Press, New York) 2002. 